Meta

Journal des traducteurs

Translators' Journal

\title{
La facture des principaux dictionnaires médicaux français : point de vue d'un traducteur
}

\section{Maurice Rouleau}

Volume 46, numéro 1, mars 2001

Traduction médicale et documentation / Medical translation and documentation

URI : https://id.erudit.org/iderudit/004003ar

DOI : https://doi.org/10.7202/004003ar

Aller au sommaire du numéro

Éditeur(s)

Les Presses de l'Université de Montréal

ISSN

0026-0452 (imprimé)

1492-1421 (numérique)

Découvrir la revue

Citer cet article

Rouleau, M. (2001). La facture des principaux dictionnaires médicaux français : point de vue d'un traducteur. Meta, 46(1), 34-55.

https://doi.org/10.7202/004003ar
Résumé de l'article

L'auteur se demande si les attentes d'un utilisateur peuvent être les mêmes à l'égard de tout dictionnaire. Pour y répondre, il compare la facture de deux dictionnaires de langue française (Le Nouveau Petit Robert et Le Petit Larousse) avec celle des quatre principaux dictionnaires médicaux utilisés par le traducteur médical. L'auteur examine les corpus de ces dictionnaires, de même que les différents éléments qui constituent une entrée type des dictionnaires médicaux et en fait une évaluation critique. 


\title{
La facture des principaux dictionnaires médicaux français: point de vue d'un traducteur
}

\author{
MAURICE ROULEAU \\ Université du Québec à Trois-Rivières, \\ Trois-Rivières, Canada
}

\begin{abstract}
RÉSUMÉ
L'auteur se demande si les attentes d'un utilisateur peuvent être les mêmes à l'égard de tout dictionnaire. Pour y répondre, il compare la facture de deux dictionnaires de langue française (Le Nouveau Petit Robert et Le Petit Larousse) avec celle des quatre principaux dictionnaires médicaux utilisés par le traducteur médical. L'auteur examine les corpus de ces dictionnaires, de même que les différents éléments qui constituent une entrée type des dictionnaires médicaux et en fait une évaluation critique.
\end{abstract}

\begin{abstract}
Are the user's expectations the same with regard to all dictionaries? To address this issue, the author compares two general French language dictionaries (The Nouveau Petit Robert and The Petit Larousse) with the four main medical dictionaries used by medical translators. He proposes a critical analysis of the corpus of these dictionaries as well as the various components of a typical entry.
\end{abstract}

\section{MOTS-CLÉS/KEYWORDS}

dictionnare médical, lexie complexe, documentation médicale, évaluation de dictionnaires, entrée type de dictionnaire

Voilà quelques années, des étudiants qui avaient à traduire un texte sur le métabolisme des médicaments n'osèrent pas rendre le verbe to metabolize par «métaboliser». La raison en était fort simple: ce verbe ne figurait alors dans aucun dictionnaire, ni général ni médical.

Plus tard, un de ces étudiants, travaillant à la pige pour une société pharmaceutique, «osa» traduire in neutropenic patients et in non-neutropenic patients par "chez le patient neutropénique » et "chez le patient non neutropénique». Quelle ne fut pas sa surprise de constater que le réviseur ${ }^{1}$ était intervenu! «Neutropénique» était devenu «en neutropénie»; «non neutropénique», «qui n’est pas en neutropénie»! La raison en était fort simple: cet «adjectif» ne figurait supposément dans aucun dictionnaire, ni général ${ }^{2}$ ni médical.

L'attente des premiers (y trouver le verbe «métaboliser») et l'attente du second (y trouver l'adjectif «neutropénique») étaient-elles justifiées? Est-ce que le fait qu'un terme ne figure pas dans un dictionnaire signifie obligatoirement que ce terme ne peut être utilisé sans risque se faire accuser de recourir à un barbarisme ou un anglicisme; ou encore que ce terme n'existe tout simplement pas? C'est se demander si un dictionnaire est nécessairement complet. Que faire alors de l'«usage» dans un domaine de spécialité comme la médecine?

Meta, XLVI, 1, 2001 
La problématique soulevée tourne autour de la facture des dictionnaires qui, elle, a plusieurs facettes: le nombre de termes, le choix des termes, les critères d'inclusion et d'exclusion d'un terme ou d'un mot, le public visé, la composition de l'équipe de rédaction, etc. Comme ces informations, qui se trouvent généralement dans les notes liminaires, dans la préface ou l'avant-propos, se font plutôt rares dans les principaux dictionnaires médicaux français, il a fallu les débusquer dans le corps même des ouvrages abordés.

L'examen de la facture des principaux dictionnaires médicaux se fera en deux temps. D'abord, nous comparerons la macrostructure des dictionnaires médicaux avec celle des dictionnaires de langue générale afin de vérifier si les attentes du lecteur à l'égard de ces deux types de dictionnaires peuvent être les mêmes. Ensuite, nous examinerons et comparerons entre eux les différents dictionnaires médicaux de langue française généralement utilisés par les traducteurs, en prenant occasionnellement comme point de référence les dictionnaires de langue.

\section{MATÉRIEL ET MÉTHODES}

\subsection{Ouvrages étudiés}

L'analyse a porté sur deux dictionnaires de langue et quatre dictionnaires médicaux, tous de langue française.

Les dictionnaires de langue retenus sont le Nouveau Petit Robert, dictionnaire privilégié par les étudiants inscrits dans un programme de traduction ou fortement suggéré par leurs professeurs, et Le Petit Larousse de l'an 2000, dictionnaire qui est très recherché ${ }^{3}$.

Des quatre dictionnaires médicaux retenus, trois sont régulièrement revus et augmentés. Le quatrième, le Dictionnaire français de médecine et de biologie, qui n’a jamais été réédité depuis sa parution en 1970-1975, a été utilisé parce qu'il est, pour ainsi dire, l'ancêtre du Dictionnaire médical de Manuila et al. (1999). Ces dictionnaires ont été choisis parce qu'ils sont d'un prix abordable, dans trois cas, et que ce sont ceux que consultent généralement les traducteurs médicaux.

Ces différents ouvrages, ainsi que leurs abréviations utilisées dans le texte, sont présentés au tableau 1.

\subsection{Sélection des pages analysées}

Comme $\mathrm{N}=30$ définit, statistiquement, une grande population, chacun des dictionnaires médicaux (PManu, GManu, Flamm, GarDe) a vu son nombre total de pages divisé par 30. Dans chacune de ces 30 tranches, deux pages ont été choisies arbitrairement: celles qui portent les numéros correspondant au premier et au second tiers de chacune des tranches. Ainsi, pour chaque dictionnaire, 60 pages (deux par tranche, une seule aurait statistiquement suffi) ont été dépouillées; ce nombre de pages a été choisi dans le but d'améliorer la précision des valeurs moyennes calculées.

L'amélioration escomptée, normalement décelable par une diminution de la valeur de l'écart type, ne s'est toutefois pas matérialisée. À preuve, les valeurs (moyenne et écart-type) obtenues avec un échantillon de 30 pages $(24,4 \pm 11,7)$ ne diffèrent pas de façon significative des valeurs obtenues avec 60 pages $(24,2 \pm 12,4)$. 


\section{RÉSULTATS}

\subsection{Analyse de données brutes}

Pour chacun des dictionnaires, le nombre d'entrées, tous genres confondus, sur chaque page choisie au hasard, a été calculé, de même que la moyenne et l'écart type des 60 valeurs. Les données sont présentées au tableau 2.

\subsubsection{Variabilité du nombre d'entrées par page}

La variabilité observée dans le nombre d'entrées par page (voir le tableau 2) est, dans certains cas, non négligeable: dans le NPRob, l'écart type est de \pm 10 (écart: 3-49);

dans le GManu, il est de de \pm 11 (écart: 16-64).

Cette variabilité s'explique différemment selon que l'ouvrage en est un de langue générale ou de médecine.

Dans sa tâche de rendre compte de la langue, un dictionnaire de langue, que ce soit le NPRob ou le Larou, doit tenir compte du phénomène de la polysémie des mots. C'est ainsi, par exemple, que l'entrée « jeu» couvre plus d'une page du NPRob, et que, à la plupart des mots commençant par «hydro-», les articles couvrent quelques lignes seulement.

Dans le domaine médical, comme probablement dans tous les domaines de spécialité, la polysémie est très rare: le spécialiste privilégie le rapport un terme/une notion. La variabilité dans les dictionnaires médicaux s'explique donc différemment.

Deux facteurs peuvent être pris en considération. Le premier est le caractère encyclopédique de ces dictionnaires. Le lecteur y cherche et y trouve plus que la simple définition du terme. Ces ajouts peuvent prendre différentes formes, selon les informations que les éditeurs sentent le besoin de fournir. C'est ainsi que le terme hémoglobine et ses différentes formes (regroupés en une ou plusieurs entrées) couvrent presque une page et demie dans le Flamm, un cinquième de page dans le GarDe, un sixième de page dans le PManu et une page et demie dans le GManu. Dans d'autres cas, l'ajout consiste en une classification, couramment utilisée pour décrire le terme vedette. C'est le cas de "lymphome» dans le Flamm ou de «lymphosarcome» dans le GarDe. De tels ajouts encyclopédiques ont, pour effet évident, de diminuer le nombre d'entrées par page.

Le second facteur est la présence de vrais synonymes, phénomène qui ne se rencontre presque pas dans la langue générale. À preuve, dans le Flamm, aux entrées «metæconémose» et «strongylose», le lecteur ne trouve rien d'autre qu'un renvoi à l'entrée principale "métæconématose», où l'existence de ces deux synonymes est signalée. La nécessité de prévoir une entrée pour chaque synonyme a pour effet d'augmenter le nombre d'entrées par page, mais non celui des termes définis.

L'importance de ce phénomène n'est pas négligeable. Une évaluation sommaire faite sur les 10 premières pages dépouillées dans les quatre dictionnaires médicaux à l'étude est révélatrice. Sur les 40 pages évaluées, une seule (dans le Flamm) ne contenait aucun renvoi (voir le tableau 3). Grosso modo, il y aurait environ $20 \%$ des entrées qui ne contiendraient pas de définition, mais seulement des renvois. Le nombre réel de «termes définis » dans chacun des dictionnaires est donc bien inférieur à celui qui est annoncé. 


\subsubsection{Nombre réel de termes dans un dictionnaire}

Le GarDe annonce 30000 «entrées», le Flamm 20000 ; le PManu parle plutôt de 26000 «termes définis» et le GManu de 150 000. Ces nombres ne sont évidemment toujours que des approximations au millier près. La différence de terminologie utilisée, tantôt « entrées", tantôt «termes définis ", soulève la question du nombre réel de termes dans un dictionnaire, surtout en regard du nombre de renvois dont il a été question précédemment.

En multipliant le nombre moyen d'entrées par page par le nombre réel de pages dans un dictionnaire, il est possible de connaitre avec assez de précision le nombre réel d'entrées dans un dictionnaire. Les résultats, présentés au tableau 2, laissent voir une grande variabilité.

Pour les dictionnaires de langue, le calcul n'est possible que pour le NPRob, version papier et version cédérom. Dans les deux cas, le pourcentage est de l'ordre de $93 \%$. Par ailleurs, vu que la version cédérom du NPRob contient 56303 entrées et que le calcul l'estime à 55755 , le pourcentage réel grimpe à 99,1\%. Le calcul utilisé permet donc d'avoir une estimation très proche du nombre réel d'entrées dans un dictionnaire.

Pour les dictionnaires médicaux, les pourcentages calculés varient de $51 \%$ à $93 \%$. Les seuls dictionnaires à annoncer un nombre d'entrées proche de la réalité sont le Flamm (avec 92,7 \%) et le GarDe (avec 89 \%). Pour les deux autres dictionnaires, le GManu et le PManu, les écarts observés sont difficilement explicables, d'autant plus que ce sont les deux seuls à parler de «termes définis" pour décrire leur contenu et que, dans les faits, il y aurait environ $20 \%$ des entrées qui ne seraient pas définies, qui ne seraient que des renvois (voir le tableau 3).

\subsection{Comparaison des dictionnaires de langue et des dictionnaires médicaux}

Un éditeur ne s'aventure à rédiger un dictionnaire que si le produit qu'il veut mettre sur le marché se distingue des produits concurrents. Cette force du marché s'applique d'autant plus aux dictionnaires spécialisés que la clientèle visée est réduite. Chaque dictionnaire, aussi bien de langue que de spécialité, devrait donc avoir sa personnalité propre, ses traits caractéristiques.

\subsubsection{Dictionnaires de langue}

Le NPRob se distingue du Larou par son caractère analogique et par son utilisation importante de citations presque essentiellement littéraires. Par exemple, à «obsession », le NPRob fournit à l'acception courante — idée, image qui s'impose à l'esprit de façon répétée et incoercible - trois exemples d'utilisation dont deux sont des citations d'auteurs. Dans le Larou, il n'y a ni exemple d'utilisation ni citation. Le NPRob, toujours à la même entrée, envoie son lecteur à d'autres termes: hantise, idée (fixe), obsessif, manie, fidèle en cela à sa mission d'être un dictionnaire analogique. Dans le Larou, il n'y a aucun renvoi.

Par contre, le Larou se distingue du NPRob par la présence d'une section de noms propres et celle de ses fameuses pages roses, sans oublier ses nombreuses illustrations. 
Il n'y a pas que des différences, il y a aussi des ressemblances. Il suffit de comparer les pages liminaires et les annexes pour s'en convaincre.

\subsubsection{Dictionnaires médicaux}

La différence entre les dictionnaires médicaux examinés dans cette étude est moins apparente que celle des dictionnaires de langue. Tous ont un caractère encyclopédique, mais chacun s'est trouvé une niche qui lui est propre. Le GarDe est le seul à offrir au lecteur des planches anatomiques et un lexique de médicaments en annexe. Le GManu et, à un degré moindre, le PManu fournissent des notes linguistiques, «qui complètent certaines définitions et qui donnent des renseignements intéressants et souvents inédits sur l'origine des termes, l'usage correct ou abusif, les décisions terminologiques des instances nationales et internationales compétentes [...]». Ils s'affichent d'ailleurs comme des dictionnaires linguistiques français de la médecine. Le GManu offre également en annexes, entre autres, un index des patronymes et un index des mots-clés du dictionnaire. Le Flamm, quant à lui, se veut, selon les mots mêmes de Jean Hamburger, le «guide dans le fatras actuel» qui caractérise la langue médicale. Il se veut utile "pour le médecin désireux de voir clair dans sa langue» et efficace «dans la critique constructive de cette langue».

Ces dictionnaires n'ont pas que des différences. Il suffit, là aussi, de comparer les pages liminaires et les annexes pour s'en rendre compte.

\subsection{Comparaison des corpus des divers dictionnaires}

Les deux dictionnaires de langue retenus pour cette étude, le NPRob et le Larou, contiennent, à peu de chose près, le même nombre d'entrées. Il en est de même pour les dictionnaires médicaux, exception faite du GManu. Le fait que le contenu soit quantitativement du même ordre de grandeur ne révèle rien de la nature même du contenu indexé, autrement dit du corpus. Le lecteur devrait-il s'attendre à trouver un contenu à peu près identique dans les différents dictionnaires généraux ou spécialisés?

\subsubsection{Contenu}

Selon le Grand Robert de la langue française (p. xxII), il faut compter entre 40000 et 70000 mots dans un dictionnaire de langue pour répondre aux besoins d'un lecteur cultivé. Il n'y a donc pas à s'étonner que le NPRob en contienne 60 000. Comme le Larou en contient autant, il y a lieu de se demander si les deux contenus s'apparentent, si les deux contenus servent aussi bien le lecteur. À titre indicatif, la liste des entrées (de «massage» à «mastic») dans le NPRob a été comparée à celle du Larou (voir l'annexe 1). Au total, 28 termes ont été relevés, dont 5 seulement ne figuraient que dans un des deux ouvrages. Les termes inscrits sont donc majoritairement ( $82 \%)$ les mêmes dans ces deux dictionnaires de langue. Le lecteur est aussi bien servi par un dictionnaire que par l'autre.

La situation est bien différente dans les dictionnaires médicaux. Le nombre total de termes compris entre «massage» et "mastologue» dans les trois dictionnaires (sauf le GManu) est de 45. De ce nombre, seulement 9, soit $20 \%$, se retrouvent dans les trois dictionnaires en question; seulement 10, dans deux dictionnaires. Quant aux 
26 autres (60\%), ils ne figurent que dans un seul des trois dictionnaires. Le lecteur n'est donc pas servi de la même façon d'un dictionnaire médical à l'autre. Les dictionnaires médicaux ne sont donc pas interchangeables.

\subsubsection{Critères d'inclusion/d'exclusion d'un terme dans un dictionnaire}

La différence dans les termes indexés, qui vient d'être notée, soulève le problème des critères d'inclusion/d'exclusion d'un terme dans un dictionnaire.

Le problème s'est posé, de façon évidente, quand A. Manuila et ses collaborateurs ont décidé de «reprendre en abrégé le Dictionnaire français de médecine et de biologie», c'est-à-dire remplacer le GManu par le PManu, «pour répondre aux besoins des personnels médicaux et paramédicaux». Les 150000 termes du GManu sont devenus 26000 termes dans le PManu. Les auteurs justifiaient leur entreprise en ces termes: «En schématisant, on peut dire qu'ils [les médecins] ne sont que rarement confrontés aux quelque 150000 termes qui rendent la communication spécialisée confuse. En revanche, ils se trouvent devant la nécessité urgente de maîtriser un vocabulaire de quelque 25000 termes qui forment le langage médical courant [...]» (PManu, p. VIII; c'est nous qui soulignons).

Dans le GManu, entre «massage cardiaque » et «mastologue», à savoir les deux termes limites utilisés pour examiner sommairement la ressemblance des entrées (voir l'annexe 1), il y a 79 termes, qui ont été ramenés à 25 dans le PManu. De ces 79 termes, une bonne douzaine pouvait être éliminée sans difficulté, car ils relevaient plus de la chimie et de la physique que de la médecine : par exemple, masse atomique, masse critique, masse au repos de l'électron, masse volumique, etc. Par contre, des 6 termes de nature réellement médicale commençant par "massif», deux seuls ont été retenus dans le PManu. Des 13 termes formés de «mastite» indexés dans le GManu, un seul a été retenu dans le PManu. À défaut de critères précis, il est difficile de justifier l'inclusion ou l'exclusion d'un terme dans un dictionnaire médical. Le seul élément de réponse serait peut-être que les termes enlevés ne font pas partie du langage médical courant. Mais comment juger du caractère courant d'un terme dans le domaine médical? Comment expliquer la différence de contenu entre les dictionnaires médicaux si ces derniers visent le même objectif? De toute évidence, la notion même de «langage médical courant» ne fait pas l'unanimité.

Une nouvelle édition s'accompagne nécessairement d'un changement plus ou moins important. Certaines définitions seront précisées, certains termes feront leur apparition en raison de l'évolution de la médecine, d'autres disparaîtront. Il est impossible de savoir quels sont les termes enlevés et les termes ajoutés. Tout au plus indique-t-on les grands domaines auxquels appartiennent les nouveaux termes. C'est ainsi que, pour la huitième édition du PManu, les nouveaux termes relèvent de l'immunopathologie, de la génétique, de l'endocrinologie, de la dermatologie, de la rhumatologie, etc. Le Flamm, dans sa sixième édition, ajoute des termes de biologie cellulaire et moléculaire, de pharmacologie, de thérapeutique, etc. Les domaines privilégiés varient donc d'un dictionnaire à l'autre, d'un éditeur à l'autre. Devant cet état de fait, le traducteur en est réduit à fouiller dans chaque dictionnaire pour trouver la notion qu'il veut connaitre. 


\subsubsection{Variété dans les catégories grammaticales des termes d'un corpus}

Comme cela vient d'être montré, les termes varient d'un dictionnaire à l'autre et les critères d'inclusion/d'exclusion ne sont jamais indiqués. Mais de quelle nature sont les termes retenus par les éditeurs? À quelle catégorie grammaticale appartiennent-ils? Comment se comparent, à cet égard, les dictionnaires de langue et les dictionnaires de médecine?

L'utilisation de la version cédérom du NPRob permet d'avoir une idée exacte de la répartition des mots (selon leur catégorie grammaticale) qui forment l'ensemble du dictionnaire. Les résultats, qui concernent les 56303 entrées recensées, sont présentés dans le tableau 4, de même que ceux du Larou. Dans ce dernier cas, la répartition est basée sur les entrées provenant de 66 colonnes choisies au hasard. Le nombre d'entrées considérées dans ce calcul est de 1365 (voir le tableau 1). Les résultats obtenus sont, à peu de chose près, identiques, preuve de la validité de l'approche statistique utilisée. Il est donc possible de dire que ces deux corpus sont tout à fait comparables en ce qui concerne la nature des mots indexés.

Le lecteur y trouve tous les mots nécessaires pour s'exprimer en français, car toutes les catégories de mots y sont représentées, dans des proportions qui reflètent leur utilité dans la formulation d'un discours cohérent. Près de $96 \%$ des entrées se trouvent dans les trois premières catégories; la quatrième, constituée par les adverbes, sans être aussi importante que les trois premières, l'est tout de même plus que les cinq dernières.

Dans le cas des dictionnaires médicaux, la situation est toute autre. Les résultats sont variables. Dans la catégorie «Noms», les valeurs varient de $74 \%$ à $94 \%$; celles des «Adjectifs", de $4 \%$ à $20 \%$; celles correspondant à «Autres» varient de $1 \%$ à $4 \%$.

La grande différence observée se situe dans la catégorie «Adjectifs». Le PManu se distingue de ses concurrents par sa grande proportion d'adjectifs $(20 \%$, cinq fois plus que dans le Flamm). Cette forte proportion est toutefois bien relative. Par exemple, entre "paranoïa» et "parasympathomimétique» (voir l'annexe 2), il y a, dans le PManu, 10 entrées qui sont des adjectifs; dans le GarDe, il y en a 9 et dans le Flamm, seulement 4. Dans le GManu, par contre, il y en a 29, même si le pourcentage d'adjectifs dans cet ouvrage n'y est que de $5 \%$.

Les résultats sont non seulement variables, mais également différents. Dans les dictionnaires médicaux, la répartition selon les catégories grammaticales est bien différente de celle relevée dans un dictionnaire de langue (voir le tableau 4). C'est dans les deux premières catégories (Noms et Adjectifs) que se trouvent près de $98 \%$ des entrées; les verbes, qui constituent $11 \%$ des mots d'un dictionnaire de langue (voir le tableau 4), sont presque inexistants dans les dictionnaires médicaux. Les autres catégories, elles, n'existent tout simplement pas. Il n'y a là rien d'étonnant quand on sait la place qu'occupe la terminologie dans un domaine de spécialité. C'est donc dire qu'un dictionnaire spécialisé en médecine n'est pas un dictionnaire de langue médicale - ce que d'ailleurs aucun ne prétend être - mais bien un dictionnaire de termes médicaux.

\section{4. Étude de l'article type}

Le contenu de l'article type varie d'un dictionnaire à l'autre, que ce dernier soit un dictionnaire de langue ou de médecine. Seul le NPRob fournit la date d'apparition 
du mot, les contraires, la transcription phonétique de toutes les entrées (le Larou ne le fait que pour les mots qui posent problème).

Contrairement au dictionnaire de langue, les dictionnaires médicaux ne fournissent aucune transcription phonétique. Il est donc impossible d'y apprendre, par exemple, comment prononcer œedématié, œdème, œsophage ou encore trichine. Ce sont donc des dictionnaires qui s'intéressent à la forme écrite et non à la forme orale des termes. Malgré cette orientation, le lecteur n'y trouvera aucun exemple d'utilisation des termes, ce que fournit généralement un dictionnaire de langue.

Dans un dictionnaire médical, le lecteur peut s'attendre, selon l'ouvrage consulté, à trouver, après l'entrée et le genre grammatical, la DCI (dénomination commune internationale), la NA (nomina anatomica), l'abréviation du terme, son origine (étymologique ou autre), l'équivalent anglais du terme, le(s) synonyme(s), la définition du terme, un développement encyclopédique et des renvois à d'autres articles complémentaires.

\subsubsection{Entrée}

Entre un dictionnaire de langue générale et un dictionnaire médical, il existe, au niveau des entrées, une différence majeure. Dans un dictionnaire de langue, l'entrée ne consiste généralement qu'en un mot (nom, adjectif, adverbe, verbe, etc.), quand elle en comprend plusieurs, ces mots sont généralement réunis par un trait d'union (par exemple, sur-le-champ $)^{4}$; dans le dictionnaire médical, quel qu'il soit, le lecteur y trouvera des «termes composés».

La présence de termes composés est quantitativement importante. La répartition des «Noms» en lexies simples (par exemple, chéiloplastie) et en lexies complexes (par exemple, convulsion clonique) est indiquée au tableau 6. Les pourcentages sont exprimés en fonction du nombre total de noms $(\mathrm{N})$ relevés dans les 60 pages examinées dans chaque dictionnaire.

Dans le Flamm et dans le GarDe, les pourcentages de lexies simples et de lexies complexes sont du même ordre de grandeur, à savoir $50 \%$. Dans les Manuila, tel n'est pas le cas. Dans le GManu, le nombre de lexies complexes est environ deux fois et demie plus élévé que celui des lexies simples; dans le PManu, c'est l'inverse.

Les auteurs des GManu et PManu étant les mêmes, ils ont donc, dans la version abrégée (PManu), décidé d'éliminer un grand nombre de lexies complexes, mais les critères utilisés pour réaliser cette opération ne sont pas précisés. Toutefois, un examen attentif de ces deux ouvrages permet d'avancer une explication, qui n'est sans doute pas la seule.

Au moment de la rédaction du GManu (1970-1975), les auteurs ont senti le besoin d'y inclure tous les synonymes connus, qu'ils soient rares ou désuets. Une telle décision entraîne forcément l'inscription de tous ces termes synonymiques comme entrées, si l'on veut que le lecteur puisse savoir le statut fonctionnel de chacun de ces termes. Prenons deux exemples: «blastomycose sud-américaine» et «blastomycose nord-américaine».

Dans le GManu, «blastomycose sud-américaine» se voit attribuer 11 synonymes; et «blastomycose nord-américaine», 6 synonymes. Dans le PManu, il n’y a qu'une seule entrée: «blastomycose». Les 19 entrées (principales et synonymiques) du GManu ont donc été ramenées à une seule dans le PManu. 
Si ce nombre d'entrées dans le GManu est comparé à celui des deux autres dictionnaires médicaux à l'étude, la différence inscrite au tableau 6 s'expliquerait également. Au lieu de 11 synonymes de «blastomycose sud-américaine», le Flamm en donne 3 et le GarDe, 4. Pour ce qui est de «blastomycose nord-américaine», au lieu des 6 synonymes indiqués dans le GManu, le Flamm en donne 2 et le GarDe, un seul. La presque totalité des synonymes étant des lexies complexes, le nombre de synonymes inscrits pourrait expliquer la très grande proportion de lexies complexes dans le GManu.

\subsubsection{Classement des lexies complexes}

La présence de lexies complexes comme entrées dans un dictionnaire pose le problème de leur classement, car leur structure est variée. Il peut s'agir d'un nom suivi d'au moins un adjectif (convulsion clonique, hépatite chronique auto-immune); d'un nom et d'un adjectif au pluriel (nerfs crâniens); d'un nom + préposition + nom commun (naissance avant terme, anémie des mineurs) ou encore d'un nom + préposition + nom propre (îlots de Langerhans, opération de Pólya). Par exemple, où trouver "réaction concertée ", sous $\mathrm{R}$ ou sous $\mathrm{C}$ ? Où trouver "fièvre de la vallée ", sous $\mathrm{V}$ ou sous $\mathrm{F}$ ? Si c'est sous $\mathrm{F}$, doit-on tenir compte des prépositions et des articles? Et où trouver "méthode de Künstcher ", sous $\mathrm{M}$ ou sous $\mathrm{K}$ ? Tout dépend du dictionnaire, sans pour autant que les principes de classement, quand ils sont indiqués, soient rigoureusement respectés. Il va sans dire que la facilité de consultation de l'ouvrage s'en trouve directement affectée.

Dans ses «Conseils pratiques", le Flamm précise que "l'indexation des termes composés ou des expressions communes se trouve au mot-clef, par exemple: thrombocytaire (série) chaînes lourdes (maladie des). Lorsque le mot-clef peut ne pas paraître évident, un renvoi figure au deuxième terme auquel le lecteur sera susceptible de se reporter. D’une manière générale, les expressions et termes éponymiques sont indexés au nom propre.» Une telle formulation laisse la porte ouverte à toutes les possibilités, car le lecteur ne peut se substituer à celui qui a établi que le mot-clef était évident. Dans le Flamm, le lecteur trouvera «hormones thymiques» à la lettre $\mathrm{H}$, mais «hormones thyroïdiennes» à la lettre $\mathrm{T}$ car, dans cette lexie, il y aurait un motclef: thyroïdiennes. Le même principe s'appliquerait à: "concertée (réaction)»! Le GarDe ne fournit aucune précision concernant la classement des termes composés d'un substantif et d'un adjectif; n'empêche que le lecteur y trouvera «nerf trijumeau» sous $\mathrm{N}$ et «nerf vague» sous V! Dans le GManu comme dans le PManu, l'ordre de présentation est l'ordre canonique (subst. + adj.).

Dans le cas où un terme contient une préposition, le GarDe a décidé de ne pas tenir compte de la préposition. C'est ainsi que, par exemple, «cellule de Betz» figure avant «cellule blastique». Le GManu a, lui aussi, opté pour cette façon de faire: le lecteur y trouvera donc «cellule en raquette» avant «cellule de Reed». Dans le PManu, il est difficile de se prononcer, car on y trouve «syndrome d'écrasement » avant «syndrome dysimmunitaire acquis (la préposition est prise en considération), mais aussi «syndrome immunodéficitaire acquis» avant "syndrome d'immunodépression T épidémique» (la préposition n'est pas prise en considération). Quant au Flamm, il tient compte de la préposition: «hospitalisation à domicile» vient avant «hospitalisation sans consentement $»$. 
Quand une entrée contient un nom propre, les façons de procéder varient également non seulement d'un dictionnaire à l'autre, mais également dans un même dictionnaire. Dans le GManu, tous les termes sont classés par ordre alphabétique des génériques (d'où la nécessité de l'index des patronymes et celui des mots-clés). Cette façon de faire a été modifiée dans le PManu. Les entrées contenant un nom propre se trouvent par ordre alphabétique des noms propres; il faut donc chercher «vaccin de Salk» à "vaccin» dans le GManu et à «Salk» dans le PManu. Les auteurs de ce dernier voulaient peut-être s'aligner sur la façon de faire du Flamm et du GarDe, qui utilisent le nom propre comme mot vedette de l'entrée.

Dans sa note au lecteur le GarDe ajoute que, si le nom propre en est un de lieu (essentiellement dans le cas des fièvres et des virus), le terme est classé sous le générique; que s'il est associé à un nom de personne, il faut le chercher au nom propre. C'est ainsi que le lecteur trouvera «virus Machupo» (Machupo: rivière bolivienne) ou «virus de Sendai (Sendai : ville du Japon)» à «virus». Cependant, la présence de «sarcome de Rous» à «sarcome» s'explique difficilement, car le nom propre désigne Francis P. Rous, un pathologiste américain. Il en est de même pour «nanisme type Fuhrman», qui figure sous N.

Dans le Flamm, les directives sont plus vagues: «D’une manière générale, les expressions et termes éponymiques sont indexés au nom propre. $\mathrm{Si}$, pour une raison précise (regroupement), il paraît préférable de mettre certaines informations sous un même générique, un renvoi figure au nom propre.» La raison invoquée pourrait s'appliquer dans le cas des fièvres et des virus, comme cela était le cas dans le GarDe. Ainsi pourrait s'expliquer la présence des fièvres comportant un nom propre, comme fièvre de Fort-Bragg, sous le générique "fièvre». Par ailleurs, il est difficilement explicable de trouver "nerf d'Arnold» à Arnold et "canalicule innominé d'Arnold» à canalicule. Dans les deux cas, il s'agit d'un nom de personne!

Quand une «entrée porte la marque du pluriel», il y a là aussi un problème de classement. Dans le GManu, on ne tient pas compte de la forme plurielle dans le classement. C'est ainsi que "cellules mastoïdiennes» figure avant «cellule médullaire». Dans le PManu, la façon de procéder a été changée: "cellule tueuse» vient avant "cellules mastoïdiennes»; "ligne médioclaviculaire», avant «lignes de croissance». Toutefois, le lecteur trouvera, dans ce même dictionnaire, "nerfs crâniens » avant «nerf récurrent»! Le Flamm et le GarDe ont, eux aussi, décidé de tenir compte de la marque du pluriel. C'est dire que les entrées commençant par «cellules» se trouvent toutes après les entrées «cellule».

\subsubsection{Nomina anatomica}

Devant le foisonnement des termes utilisés pour décrire une même structure anatomique, les anatomistes ont décidé, en 1895, d'en uniformiser la nomenclature. Pour éviter tout soubresaut nationaliste, ce fut la langue latine qui a été retenue comme langue officielle de cette terminologie. La Nomina anatomica (NA), comme elle s'appelle, a été francisée par le Collège médical français des professeurs d'anatomie. La sixième et dernière édition ${ }^{5}$ de cette nomenclature date de 1989.

Même si, dans le GManu, la NA figurait, les auteurs ont décidé de l'éliminer dans la version abrégée (PManu). Le Flamm et le GarDe fournissent la PNA (Parisiensis Nomina Anatomica) ou plus simplement la NA. Il n'est nulle part indiqué que 
le nom français utilisé comme entrée au dictionnaire est le terme francisé de la NA: dans le Flamm et le PManu, les entrées correspondant à $n$. vagus (NA) sont respectivement «nerf pneumogastrique» et "pneumogastrique», alors que la version francisée est «nerf vague». L'ancienne «valvule tricuspide» devrait s'appeler, selon la NA francisée, «valve atrio-ventriculaire droite» mais, dans le PManu et le Flamm, elle est toujours la valvule tricuspide; pire, le Flamm lui attribue même une fausse NA en l'appelant valvula atrioventricularis dextra au lieu de valva atrioventricularis dextra. Les anatomistes ont voulu rectifier l'illogisme de cette ancienne appellation. En effet, l'emploi du suffixe diminutif -ule (valvule) pour désigner un objet qui est plus gros que la valve choque le bon sens. Ce qui était valvule est devenu valve et ce qui était valve est devenu valvule. Du moins, c'est ce que les anatomistes voudraient, mais les dictionnaires ne semblent pas prêts à les suivre sur ce terrain.

\subsection{4. Étymologie}

Le Flamm et le GarDe sont les deux seuls dictionnaires à fournir l'étymologie des termes. Et dans les deux cas, le fait que les racines qui contribuent à la formation du mot ne soient pas toutes de la même langue n'est jamais signalé. Le dictionnaire où il serait le plus approprié de trouver de telles remarques serait le Flamm, qui se veut le gardien de la langue médicale. Mais ce dictionnaire ne mentionne même pas le fait (voir vasectomie, atriomégalie, bactéricide, etc.). Les auteurs semblent accepter qu'un terme ait une double origine du point de vue étymologique ${ }^{6}$.

C'est sans doute ce qui explique que, dans le Flamm, l'hybride «mammographie» soit préféré à «mastographie » comme entrée principale (c'est l'inverse dans le GarDe) ou encore que "rectotomie», un autre hybride, soit préféré à proctotomie (pour le GarDe, ces deux termes ne sont pas synonymes, car ils ont chacun leur définition). L'usage y est peut-être, inconsciemment, pour quelque chose. Mais tel n'est pas toujours le cas. Le Flamm invoque la mauvaise construction étymologique pour justifier l'emploi de «tétraplégie » à la place de «quadriplégie» (dans le GarDe, l'entrée principale est à «quadriplégie», sans plus). Dans le Flamm, il est mentionné, à «mastectomie», que «mammectomie» est incorrect bien que "couramment utilisé»; dans le GarDe, on ne fait que mentionner qu'il est incorrect. Le PManu, pour sa part, se contente de les donner comme synonymes, mais donne la définition à «mastectomie».

\subsubsection{Synonymes}

Il a été mentionné précédemment que le GManu fournit un grand nombre de synonymes mais, des synonymes, il s'en trouve dans tous les dictionnaires médicaux même si, parfois, ceux qui sont proposés ont de quoi dérouter le lecteur.

Par exemple, le Flamm donne, comme synonyme de «blastomycose», «ajellomycose », terme qui constitue également une entrée dans le dictionnaire; le GarDe, quant à lui, propose, comme synonyme du même mot, "exascose», qui ne figure dans aucun dictionnaire, même pas dans le GarDe. Quant au PManu, il ne propose aucun synonyme.

L'autre problème que pose l'existence des synonymes, c'est de savoir sous quel terme se trouve la définition. Sous le terme le plus couramment utilisé? Tel n'est pas 
toujours le cas; il suffit de se rappeler ce que dit le Flamm à «mastectomie» (voir ci-dessus à «Étymologie»). Cela est peut-être compréhensible dans le cas des mots hybrides mais, dans le cas plus courant des synonymes, le lecteur est simplement renvoyé d'une entrée à l'autre. Dans le cas de la paire «gonadotrophine/ gonadostimuline», l'entrée principale varie selon le dictionnaire. Dans le Flamm, la définition se trouve à «gonadotrophine»; dans le Garde, à «gonadostimuline». Un autre exemple: civière/brancard. Le NPRob ne les donne pas comme synonymes mais, dans les dictionnaires médicaux, ils sont considérés comme tels. Dans le GManu, la définition se trouve à «brancard»; dans le PManu, elle se trouve à «civière»; pourtant le GManu est l'ancêtre du PManu. Dans le GarDe, une seule entrée: «brancard», même si «civière» y est donné comme synonyme. Dans ces cas, le lecteur est au moins renvoyé à l'entrée principale. Mais que dire d'un renvoi à un renvoi ?

Pour qui veut savoir comment le Flamm définit le «syndrome de Reiter», il se rend d'abord à "Reiter (syndrome de)", s'il sait que les termes éponymiques sont généralement classés par nom propre. Là, on l'envoie à «Feissinger-Leroy-Reiter (syndrome de)», pour y apprendre qu'il doit aller voir «oculo-urétro-synovial (syndrome)». Le lecteur s'y rend, avec l'espoir secret, d'y trouver enfin ce qu'il cherche désespérément : la définition du terme. Dans le GarDe, les cinq synonymes mentionnés renvoient tous à une seule et même entrée: "Fiesinger et Leroy ou FiessingerLeroy-Reiter (syndrome de Noël) ${ }^{7} »$. Cela ne signifie pas pour autant que le GarDe ne se permet pas certaines libertés: à «LH», le GarDe envoie le lecteur à "gonadotrophine» pour se faire dire que la définition se trouve à "gonadostimuline» (le Flamm lui, a décidé de fournir la définition à $\mathrm{LH}$ ). Quand on sait que le temps est la denrée qui manque le plus souvent au traducteur, le problème de ces renvois prend de l'importance.

\subsection{6. Équivalents anglais}

Des quatre dictionnaires médicaux à l'étude, un seul, le GManu, ne fournit pas d'équivalents anglais. Pourquoi, dans des dictionnaires qui ne se veulent pas bilingues, fournir des équivalents anglais ou de traductions anglaises, pour reprendre l'expression utilisée par certains dictionnaires? La réponse est probablement celle que fournit le PManu dans l'avant-propos de la huitième édition: "faciliter l'accès à la littérature anglo-saxonne». Il n'est nulle part précisé ce que les éditeurs ou les rédacteurs des trois dictionnaires entendent par langue anglo-saxonne. Il y a fort à parier que la langue anglaise américaine en fait partie car, s'il est un endroit où se publient nombre d'articles et de livres médicaux, c'est bien aux États-Unis.

Le lecteur devrait donc s'attendre à trouver les équivalents anglais proposés dans les principaux dictionnaires médicaux anglais (le Dorland's ou encore le IDMB). Tel n'est pas toujours le cas. Hematosis, équivalent anglais proposé pour «hématose» par les trois dictionnaires, ne figure dans aucun des deux dictionnaires anglais consultés. Autres exemples, dans le Flamm, «syndrome de Laron» a pour équivalent anglais Laron's syndrome; dans le GarDe, on trouve «nanisme type Laron» avec l'équivalent anglais «Laron type dwarfism». Ces deux équivalents anglais ne figurent ni dans le Dorland's ni dans le IDMB.

Il arrive également que le lecteur ne trouve aucun équivalent mentionné, sans pour autant que l'on sache pourquoi. Ce n'est pas toujours le signe d'un vide 
terminologique: dans le GarDe, à «noyau rouge (syndrome alterne du)», le lecteur ne trouve pas l'équivalent anglais Claude's syndrome, qui pourtant devrait être connu du rédacteur de cet article.

Il arrive aussi que le lecteur trouve, dans le Flamm, la mention: "pas d'équivalent connu». C'est le cas à «gonarthrose». Que veut réellement dire cette mention? Qu'il n'existe pas d'équivalent ou tout simplement que le rédacteur de l'article ne le connait pas? Dans le GarDe, deux équivalents sont pourtant proposés : gonarthosis et osteoarthritis of the knee; dans le PManu, un seul est fourni: degenerative osteoarthritis of the knee. De toute évidence, les rédacteurs de ces articles n'ont pas de la langue anglaise la même maitrise. Dans le Dorland's et le IDMB, le lecteur y trouvera facilement gonarthosis. Les deux autres équivalents proposés semblent plutôt être des traductions que des termes médicaux anglais reconnus, du moins de ce côté-ci de l'Atlantique.

\subsubsection{Définitions}

Le traducteur consulte souvent ses dictionnaires médicaux pour y connaître le sens d'un terme, car il n'est pas spécialiste en la matière. Il s'attend donc à pouvoir combler son besoin en consultant l'un ou l'autre des dictionnaires qu'il a à sa disposition. Mais tel n'est pas toujours le cas. Il arrive que des définitions soient étrangement formulées ou encore, ce qui est plus surprenant, qu'elles differrent selon le dictionnaire consulté. Le traducteur médical, devant un tel état de fait, est embarrassé.

Le lecteur apprend, dans le Flamm, que «apocrine adj.» est un «mode de sécrétion exocrine». Il n'est pas dans les règles de définir un adjectif par un substantif. On s'attendrait à trouver: "se dit d'une glande dont le produit... », formulation qui a d'ailleurs été retenue, dans ce même dictionnaire, pour définir "exocrine». Un problème de cette nature soulève la question de la pertinence d'un lexicographe dans l'équipe rédactionnelle.

Quant à l'unanimité sur un concept, elle fait défaut, non pas de façon systématique, mais assez souvent pour que le phénomène soit gênant. Il n'est pas de la compétence du traducteur de décider quelle acception doit avoir un terme. Alors, quand un tel cas se présente, il doit consulter plusieurs sources et opter pour l'acception que la majorité privilégie. Mais que dira le lecteur s'il a à sa disposition un seul dictionnaire et que la définition qu'il y trouve ne lui permet pas de comprendre correctement le texte qu'il lit?

Voici deux exemples où une certaine harmonisation entre les divers dictionnaires serait bienvenue. Le premier porte sur un terme très courant en pharmacologie: "posologie» et le second sur un terme plus spécialisé: «oxytocine».

Les définitions proposées par les principaux dictionnaires médicaux pour le terme «posologie» sont les suivantes:

Flamm: 1) Étude des doses thérapeutiques des médicaments suivant l'âge, le sexe, l'état du malade; 2) Indication figurant sur une préparation spécialisée ou sur l'ordonnance magistrale à la suite de la formulation de chaque médicament, pour en préciser le nombre de prises journalières, leur espacement et la durée d'administration.

GarDe: 1) Étude des doses thérapeutiques des divers médicaments suivant l'âge, le sexe et l'état du malade; 2) Mode d'emploi d'un médicament. - Ce terme est souvent employé incorrectement comme synonyme de dose. 
GManu: Quantité totale, estimée d'après l'âge et le poids du malade — et éventuellement renouvelée à intervalles plus ou moins longs — d'un médicament à administrer en une ou plusieurs fois pour traiter une maladie (OMS).

PManu: Quantité totale d'un médicament, estimée selon l'âge et le poids du malade, qui est à administrer en une ou plusieurs fois lors du traitement d'une maladie.

Les différences notées entre ces définitions parlent par elles-mêmes. Selon que le terme désigne l'étude, le mode d'emploi ou les indications, le traducteur devra recourir à un verbe différent s'il veut utiliser correctement ce terme en contexte.

Le second exemple est aussi révélateur, sinon plus. Voici les définitions du terme «oxytocine» trouvées dans les différents dictionnaires:

Flamm: analogue synthétique de l'ocytocine naturelle.

GarDe: [Le terme n'y figure pas $^{8}$.]

GManu: hormone polypeptidique, du lobe postérieur de l'hypophyse, de structure voisine de la vasopressine [...] Syn. alpha-hypophamine, ocytocine, pitocine.

PManu: hormone du lobe postérieur de l'hypophyse, produite par les noyaux nerveux paraventriculaires [...] Syn. Ocytocine, pitocine.

Devant ces différentes définitions, le traducteur est en droit de se demander s'il peut utiliser «oxytocine» (il ne figure pas dans le GarDe) ou, s'il l'utilise, quel sens lui donner: celui d'un produit naturel (PManu, GManu) ou d'un produit de synthèse (Flamm). Et s'il s'agit d'un produit naturel, où est-il produit: dans l'hypothalamus (GarDe, PManu) ou dans l'hypophyse (GManu) ? Seul le Flamm permet de résoudre cette difficulté. En effet, à « ocytocine», terme qui, dans ce dictionnaire, désigne le produit naturel, il est dit qu'il s'agit d'un «nonapeptide cyclique, isolé initialement du lobe postérieur de l'hypophyse (d'où le nom actuellement inexact d'hormone post-hypophysaire), élaborée par certains neurones magnocellulaires de l'hypothalamus $[\ldots] »$.

Le manque d'harmonisation entre les divers dictionnaires médicaux n'est pas rarissime, contrairement à ce qu'on pourrait croire. Pour s'en convaincre, il suffit de comparer, entre autres, les définitions fournies dans les différents dictionnaires des termes suivants: glycémie, pleurésie, hospitalisation à domicile, tympanoplastie/ myringoplastie.

\section{DISCUSSION}

Les résultats et observations présentés dans cette étude démontrent que le lecteur, qu'il soit traducteur ou non, ne peut avoir à l'égard des dictionnaires médicaux les mêmes attentes qu'il a à l'égard des dictionnaires généraux. Il s'agit en fait de deux types de dictionnaires bien différents. Le NPRob et le Larou sont réellement des dictionnaires de langue. En effet, le lecteur y trouvera tous les genres de mots nécessaires à la construction d'une phrase française (nom, pronom, article, verbe, etc.) (voir le tableau 4), tous les mots qu'un francophone cultivé est susceptible d'utiliser dans la conversation courante, presque tous ceux qu'il rencontrera dans un périodique général d'information et une grande partie de ceux qui se trouvent dans les textes littéraires, comme l'indique Le Grand Robert (p. XxII). Et à ce titre, ces deux dictionnaires généraux sont comparables (voir le tableau 2). De plus, les dictionnaires de langue offrent à tous leurs lecteurs la transcription phonétique des mots, soit à toutes les entrées (dans le NPRob) ou aux seules entrées qui posent problème 
(dans le Larou). Ces dictionnaires s'intéressent donc à la langue écrite et à la langue parlée.

Les dictionnaires médicaux, eux, ne s'intéressent qu'à la forme écrite des termes et non à leur prononciation, même si cette dernière pose des problèmes. Ce ne sont pas des dictionnaires de langue. En effet, leur contenu consiste essentiellement en noms et adjectifs (voir le tableau 5) sans que, pour autant, tous les noms et adjectifs y figurent, la preuve en étant l'absence du verbe «métaboliser» et de l'adjectif «neutropénique». Ce sont donc des dictionnaires de termes médicaux. D’ailleurs, l'un d'entre eux, le GarDe, porte bel et bien son nom: Dictionnaire des termes de médecine. Selon le PManu, les termes retenus, environ 25 000, forment «le langage ${ }^{9}$ médical courant ». Les différents éditeurs semblent s'entendre sur ce nombre, car leur contenu est du même ordre de grandeur, à l'exception du GManu, dictionnaire en quatre volumes (voir le tableau 2). Là où ils ne s'entendent pas, c'est sur la notion même de "langage médical courant».

Ce nombre de 25000 appelle deux remarques. D'abord, la réalité du nombre d'entrées ou de termes définis qu'annoncent les éditeurs. Le pourcentage de nombres réels d'entrées par rapport aux nombres déclarés est très variable (voir le tableau 2). Il va de $50 \%$, dans le cas du PManu, à $93 \%$ dans le cas du Flamm). Il y a donc lieu de se demander comment les éditeurs ont pu en arriver à cette évaluation. Ensuite, il y a la nature de ces 25000 entités. Les éditeurs parlent tantôt de termes définis (PManu, par exemple), tantôt d'entrées (Flamm, par exemple). Or, une évaluation approximative indique qu'il y a environ $20 \%$ des entrées qui sont des renvois (voir le tableau 3), c'est-à-dire des termes qui ne sont pas définis. Il faudrait donc, pour connaître le nombre réel de termes définis, réduire d'environ $20 \%$ les nombres réels d'entrées. On serait donc encore plus loin de la réalité annoncée.

La notion de «langage médical courant» ne semble pas la même pour les différents éditeurs, malgré l'affichage d'un contenu de même importance quantitative. En effet, la comparaison du contenu des dictionnaires laisse clairement voir que les termes choisis diffèrent selon le dictionnaire (voir le tableau 6 et les annexes 1 et 2); les dictionnaires de langue française analysés ont un contenu presque identique (voir l'annexe 1). Il y a alors lieu de se demander ce qui détermine le choix d'un terme à inclure ou à exclure d'un dictionnaire. Serait-ce la clientèle visée? Cet aspect serait à examiner de plus près. De ce côté-ci de l'Atlantique, ce ne sont pas les étudiants en médecine ni les médecins qui sont les plus grands consommateurs de dictionnaires médicaux. De l'autre côté de l'Atlantique, qu'en est-il ? Car ce sont eux qui devraient constituer le public visé.

Le traducteur fait partie du public visé soit nommément, comme dans le GarDe, soit implicitement, comme dans le PManu, en tant qu'utilisateur du vocabulaire médical au quotidien. Il se fait un devoir, en autant que ses moyens lui permettent, d'acheter toute nouvelle édition de son dictionnaire médical. Mais peut-il réellement se contenter d'un seul? Non, car les contenus sont loin d'être comparables. Il lui faut donc en acheter plusieurs.

Il est bien connu que le temps est ce qui fait le plus défaut au traducteur. Dans ce contexte, la consultation d'un dictionnaire se devrait d'être facile et rentable. Or, tel n'est pas le cas, la consultation pose bien des pièges au traducteur - et à tout utilisateur. Non seulement le traducteur doit-il consulter plusieurs dictionnaires dans l'espoir de trouver le terme qu'il cherche, mais encore il lui faut s'assurer, quand le 
terme apparaît dans un dictionnaire, que la définition fournie est la même dans un autre dictionnaire médical. Il n'est pas normal que le lecteur ait à décider de l'acception d'un terme. S'il consulte un dictionnaire, c'est qu'il veut connaître la définition et non être soumis à un choix de définitions.

La consultation des dictionnaires médicaux pose d'autres embûches, en raison de la surabondance de lexies complexes comme entrées (voir le tableau 6). Même la recherche d'un terme formé seulement d'un nom et d'un adjectif peut être chronophage, car les principes de classement des termes composés ne sont jamais clairement exprimés ni respectés. La présence, dans une lexie complexe, d'une préposition, d'un nom propre ou de la marque du pluriel oblige le traducteur à envisager toutes les possibilités de classement avant de décider que le terme ne figure pas dans le dictionnaire consulté, ce qui constitue une autre opération chronophage.

Les dictionnaires médicaux ne fournissent pas que la définition du terme. Ils y ajoutent, entre autres, la NA et les équivalents anglais. De toute évidence, la NA ne fait pas partie des préoccupations majeures des éditeurs. En effet, elle existait dans le GManu, mais elle a disparu du PManu. La présence de la NA n'est pas toujours assurée, et les corrections apportées dans la NA francisée (valve/valvule) ne figurent pas, sans oublier la présence de quelques erreurs dans la NA latine.

Quant à la présence des équivalents anglais, elle pose des problèmes. Même si les dictionnaires médicaux ne s'affichent pas comme des dictionnaires bilingues, ils ne manquent pas, dans leur publicité, de préciser qu'ils contiennent des équivalents anglais. L'utilité de ces équivalents est douteuse, car la maîtrise de la langue anglaise semble varier beaucoup d'un rédacteur à l'autre. Il n'est donc pas recommandé de s'en servir comme dictionnaire bilingue.

Bref, la consultation des dictionnaires médicaux par le traducteur est une opération pénible et chronophage. Le problème le plus grave est certainement la divergence des définitions. La création d'un comité d'harmonisation formé de représentants des différentes maisons d'éditions serait bienvenue, non seulement pour le traducteur, mais pour tout utilisateur d'un dictionnaire médical.

\section{NOTES}

1. La forme masculine n'est ici utilisée que pour alléger le texte.

2. En fait, «neutropénique» figurait déjà dans le Grand Robert de la langue française en 1991.

3. «Le Petit Larousse illustré 2000 a été, tous genres confondus, le livre qui s'est le mieux vendu en 1999 (1,07 million d'exemplaires), indique la revue professionnelle française Livres-Hebdo dans son numéro qui paraît aujourd'hui. » (La Presse, 14 janvier 2000)

4. Dans le Larou, deux termes composés ont été trouvés et tous deux proviennent du domaine médical: Quincke (œdème de) et Korsakoff (syndrome de); ces deux entrées ne figurent pas dans le NPRob.

5. La Nomina Anatomica a été remplacée, en 1998, par la Terminologia Anatomica. FCAT (Federative Committee on Anatomical Terminology) (1998): Terminologia Anatomica: International Anatomical Terminology, Stuttgart, New York, Thieme. Disponible également sur cédérom.

6. En ce sens, ils ne se démarquent pas du NPRob où il est dit à la page xIv : «Les mots savants sont traditionnellement formés avec des radicaux latins (octogénaire) ou grecs (stéthoscope), parfois hybrides (monocle) et autrefois critiqués par les puristes. Aujourd'hui, on va plus loin: un très grand nombre de mots mêlent le grec ou le latin au français et le modèle est de plus en plus productif (cryopréservation, hydrocarbure, etc.).»

7. La pertinence d'inclure un seul prénom, Noël, quand il y a deux ou trois noms propres dans le terme, est fort discutable. 
8. Le lecteur y trouvera par contre ocytocine défini comme suit: hormone polypeptidique sécrétée par les noyaux paraventriculaires et supra-optique de l'hypothalamus et stockée dans le lobe postérieur de l'hypophyse.

9. Les auteurs font rarement la distinction entre langue ou langage et vocabulaire, comme si la langue n'était constitué que de termes.

\section{RÉFÉRENCES}

Dorland's Illustrated Medical Dictionary (1994), 28th ed., Philadelphia, W.B. Saunders Co.

Garnier, M., V. Delamare, J. et T. Delamare (1998): Dictionnaire des termes de médecine, $25^{\mathrm{e}}$ éd., Paris, Maloine.

International Dictionary of Medicine and Biology (1986), New York, John Wiley \& Sons, 3 vol.

Kernbaum, S. (1998): Dictionnaire de médecine Flammarion, $6^{\mathrm{e}}$ éd., Paris, Flammarion, coll. «Médecine Sciences».

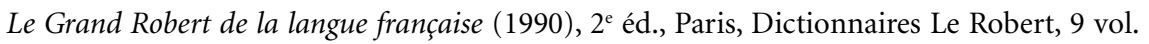

Le Nouveau Petit Robert (1996), Paris, Dictionnaires Le Robert.

Le Petit Larousse de l'an 2000, grand format, (1999), Paris, Larousse/HER.

Manuila, A. et L., M. Nicole et M. Lambert (1970-1975) : Dictionnaire français de médecine et de biologie, Paris, Masson, 4 vol.

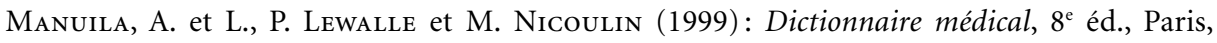
Masson.

\section{ANNEXE}

\section{TABLEAUX}

\section{Tableau 1 : Liste des dictionnaires utilisés}

\begin{tabular}{|c|c|c|}
\hline Type & Abrév. & Titre \\
\hline \multirow[t]{2}{*}{$\begin{array}{l}\text { Dictionnaire de } \\
\text { langue française }\end{array}$} & NPRob & $\begin{array}{l}\text { Le Nouveau Petit Robert (version cédérom, 1996; } \\
\text { version papier, 1996) }\end{array}$ \\
\hline & Larou & Le Petit Larousse de l'an 2000, grand format (1999) \\
\hline \multirow[t]{4}{*}{ Dictionnaire médical } & PManu & $\begin{array}{l}\text { Dictionnaire médical, }(1999) \\
\text { (communément appelé le Petit Manuila) }\end{array}$ \\
\hline & GManu & $\begin{array}{l}\text { Dictionnaire français de médecine et de biologie, } \\
\text { (1970-1975) (communément appelé le Grand Manuila) }\end{array}$ \\
\hline & Flamm & Dictionnaire de médecine Flammarion, (1998) \\
\hline & GarDe & $\begin{array}{l}\text { Le Garnier-Delamare, Dictionnaire des termes } \\
\text { de médecine (1998) }\end{array}$ \\
\hline
\end{tabular}


Tableau 2 : Données brutes

\begin{tabular}{|c|c|c|c|c|c|c|}
\hline Dictionnaire & Nombre total & $\begin{array}{l}\text { Nombre moyen } \\
\text { d'entrées/page }\end{array}$ & $\begin{array}{l}\text { Nombre réel } \\
\text { de pages }^{* *}\end{array}$ & $\begin{array}{l}\text { Nombre total } \\
\text { d'entrées } \\
\text { calculées }\end{array}$ & $\begin{array}{l}\text { Nombre total } \\
\text { d'entrées } \\
\text { annoncées }\end{array}$ & $\begin{array}{l}\text { \% du nombre } \\
\text { d'entrées } \\
\text { annoncées }\end{array}$ \\
\hline Larou $^{*}$ & 1365 & - & - & - & 59000 & \\
\hline $\begin{array}{l}\text { NPRob }^{* *} \\
\text { (cédérom) }\end{array}$ & 56303 & & & & 60000 & 93,8 \\
\hline $\begin{array}{l}\text { NPRob } \\
\text { (papier) }\end{array}$ & 1407 & $23 \pm 10$ & 2425 & $\begin{array}{r}55775 \\
\pm 24250\end{array}$ & 60000 & 93,0 \\
\hline Flamm & 1227 & $20 \pm 5$ & 927 & $\begin{array}{r}18540 \\
\pm 4635\end{array}$ & 20000 & 92,7 \\
\hline GarDe & 1889 & $31 \pm 7$ & 858 & $\begin{array}{r}26598 \\
\pm 6006\end{array}$ & 30000 & 88,7 \\
\hline GManu & 2176 & $36 \pm 11$ & 2967 & $\begin{array}{r}106812 \\
\pm 32637\end{array}$ & 150000 & 71,2 \\
\hline PManu & 1451 & $24 \pm 4$ & 551 & $\begin{array}{r}13224 \\
\pm 2204\end{array}$ & 26000 & 50,9 \\
\hline
\end{tabular}

* La présence dans ce dictionnaire de nombreuses illustrations occupant des surfaces plus ou moins importantes rend impossible le calcul du nombre de mots par page et, par conséquent, celui du nombre total de pages. Le nombre d'entrées recensées porte sur un nombre de colonnes $(\mathrm{N}=66)$ suffisant pour atteindre un total de 1000 entrées, valeur comparable à celles des autres dictionnaires.

* Certaines cases sont vides, car les données ont été obtenues directement sur le cédérom.

${ }^{* * *}$ Les nombres de pages ne correspondent pas toujours aux nombres de pages des ouvrages, car certains éditeurs ont décidé qu'une lettre ne commencerait jamais au verso d'une feuille. Le nombre de pages a donc été corrigé en conséquence.

Tableau 3: Fréquence des renvois dans les dictionnaires médicaux

\begin{tabular}{|c|c|c|c|}
\hline Dictionnaire & $\begin{array}{c}\text { Nombre moyen } \\
\text { d'entrées par page } \\
\text { (écart) }\end{array}$ & $\begin{array}{c}\text { Nombre moyen } \\
\text { de renvois par page } \\
\text { (écart) }\end{array}$ & $\begin{array}{c}\text { \% de renvois } \\
\text { par page }\end{array}$ \\
\hline Flamm & $\begin{array}{c}20,9 \pm 5,0 \\
(14-28)\end{array}$ & $\begin{array}{c}3,4 \pm 3,2 \\
(0-10)\end{array}$ & $16,3 \%$ \\
\hline GarDe & $\begin{array}{c}29,1 \pm 5,3 \\
(21-37)\end{array}$ & $\begin{array}{c}8,2 \pm 6,4 \\
(1-22)\end{array}$ & $28,2 \%$ \\
\hline GManu & $\begin{array}{c}36,4 \pm 9,2 \\
(22-47)\end{array}$ & $\begin{array}{c}7,9 \pm 4,7 \\
(3-19)\end{array}$ & $21,7 \%$ \\
\hline PManu & $26,4 \pm 4,5$ & $4,3 \pm 2,8$ & $16,3 \%$ \\
& $(20-34)$ & $(1-10)$ & \\
\hline
\end{tabular}


52 Meta, XLVI, 1, 2001

Tableau 4: Répartition des mots dans les dictionnaires généraux selon leur catégorie grammaticale

\begin{tabular}{|l|c|c|c|}
\hline Catégorie de mot & $\begin{array}{c}\text { NPRob } \\
\%\end{array}$ & $\begin{array}{c}\text { Larou } \\
\%\end{array}$ & $\begin{array}{c}\text { Valeur moyenne } \\
\%\end{array}$ \\
\hline Noms & 60,82 & 63,59 & 62,21 \\
\hline Adjectifs & 22,74 & 22,05 & 22,40 \\
\hline Verbes & 11,41 & 10,48 & 10,95 \\
\hline Adverbes & 2,97 & 2,27 & 2,62 \\
\hline Conjonctions & 0,06 & 0,07 & 0,07 \\
\hline Interjections & 0,33 & 0,29 & 0,31 \\
\hline Prépositions & 0,15 & 0,15 & 0,15 \\
\hline Pronoms & 0,13 & 0,22 & 0,18 \\
\hline Autres & 1,39 & 0,88 & 1,14 \\
\hline
\end{tabular}

Tableau 5 : Répartition des mots dans les dictionnaires médicaux selon leur catégorie grammaticale

\begin{tabular}{|l|c|c|c|c|c|}
\hline Catégorie & $\begin{array}{c}\text { Flamm } \\
\%\end{array}$ & $\begin{array}{c}\text { GarDe } \\
\%\end{array}$ & $\begin{array}{c}\text { GManu } \\
\%\end{array}$ & $\begin{array}{c}\text { PManu } \\
\%\end{array}$ & $\begin{array}{c}\text { Valeur moyenne } \\
\%\end{array}$ \\
\hline Noms & 94,05 & 88,93 & 94,07 & 73,88 & 87,73 \\
\hline Adjectifs & 3,75 & 7,31 & 5,33 & 19,71 & 9,01 \\
\hline Verbes & 0,08 & 0,0 & 0,05 & 0,28 & 0,10 \\
\hline $\begin{array}{l}\text { Adverbes, conjonctions, } \\
\text { interjections, pronoms, } \\
\text { prépositions }\end{array}$ & 0 & 0 & 0 & 0 & 0 \\
\hline Autres & 2,12 & 3,76 & 0,55 & 6,13 & 3,14 \\
\hline
\end{tabular}

Tableau 6 : Répartition des noms en lexies simples et en lexies complexes dans les dictionnaires médicaux

\begin{tabular}{|l|c|c|c|c|}
\hline Lexie & Flamm & GarDe & GManu & PManu \\
\hline Simple (\%) & $48 \%$ & $51 \%$ & $29 \%$ & $68 \%$ \\
\hline Complexe (\%) & $52 \%$ & $49 \%$ & $71 \%$ & $32 \%$ \\
\hline N & 1154 & 1680 & 2047 & 1072 \\
\hline
\end{tabular}




\section{COMPARAISON DU CONTENU DES DICTIONNAIRES À L'ÉTUDE}

Figurent en gras les termes rencontrés dans un seul des dictionnaires comparés.

A) Dans les dictionnaires de langue

\begin{tabular}{|l|l|}
\hline NPRob & Larou \\
\hline Massage & Massage \\
\hline Massaliote & Massaliote \\
\hline Masse & Masse \\
\hline Massé & \\
\hline Masselotte & Masselotte \\
\hline Massepain & Massepain \\
\hline Masser & Masser \\
\hline Massette & Massette \\
\hline Massette & Massette \\
\hline Masseur & Masseur \\
\hline Massicot & Massicot \\
\hline Massicoter & Massicoter \\
\hline & Massier \\
\hline Massif & Massif \\
\hline
\end{tabular}

\begin{tabular}{|l|l|}
\hline NPRob & Larou \\
\hline Massification & Massification \\
\hline Massifier & Massifier \\
\hline Massique & Massique \\
\hline Massivement & Massivement \\
\hline Mass media & Mass media \\
\hline & Massivité \\
\hline Massore & Massore \\
\hline Massorète & Massorète \\
\hline Massue & Massue \\
\hline Mastaba & Mastaba \\
\hline & Mastard \\
\hline Mastectomie & Mastectomie \\
\hline Mastère & \\
\hline Mastic & Mastic \\
\hline
\end{tabular}

B) Dans les dictionnaires médicaux

\begin{tabular}{|c|c|c|}
\hline PManu & Flamm & GarDe \\
\hline \multirow[t]{4}{*}{ Massage cardiaque } & Massage cardiaque & Massage cardiaque \\
\hline & Massage tubaire & \\
\hline & Masse & \\
\hline & Masse du noyau (défaut de) & \\
\hline Masse moléculaire & & Masse moléculaire \\
\hline Masse sanguine & Masse sanguine & \\
\hline Masséter & & Masséter \\
\hline \multicolumn{3}{|l|}{ Massétérin } \\
\hline & & Masseur-kinésithérapeute \\
\hline moniteur & & Masseur-kinésithérapeute \\
\hline \multicolumn{3}{|l|}{ Massif carpien } \\
\hline \multicolumn{3}{|l|}{ Massif facial osseux } \\
\hline & Massif nasal médian & \\
\hline & Massokinésithérapie & Massokinésithérapie \\
\hline & Masson (cellule claire de) & \\
\hline & Masson (col. trichrom. de) & Masson (col. trichrom. de) \\
\hline
\end{tabular}


54 Meta, XLVI, 1, 2001

\begin{tabular}{|c|c|c|}
\hline & Masson (méthode de) & \\
\hline & Massothérapie & Massothérapie \\
\hline & & Massue \\
\hline & Massue terminale de Held & \\
\hline \multicolumn{3}{|l|}{ Mast-, masto } \\
\hline & & Mastalgie \\
\hline Mastectomie & Mastectomie & Mastectomie \\
\hline \multicolumn{3}{|l|}{ Mastectomisé } \\
\hline & & Master (épreuve de) \\
\hline & Masters et Allen (syndr. de) & Masters et Allen (syndr. de) \\
\hline \multicolumn{3}{|l|}{ Masticateur } \\
\hline Mastication & Mastication & Mastication \\
\hline \multicolumn{3}{|l|}{ Masticatoire } \\
\hline \multicolumn{3}{|c|}{ Masticothérapie } \\
\hline \multirow[t]{2}{*}{ Mastite } & Mastite & Mastite \\
\hline & & Mastoblaste \\
\hline Mastocyte & Mastocyte & Mastocyte \\
\hline \multirow[t]{2}{*}{ Mastocytome } & Mastocytome & Mastocytome \\
\hline & Mastocytopexie & \\
\hline \multicolumn{3}{|l|}{ Mastocytose } \\
\hline \multicolumn{3}{|c|}{ Mastocytose maligne } \\
\hline & & Mastocytoxanthome \\
\hline Mastodynie & Mastodynie & Mastodynie \\
\hline Mastographie & Mastographie & Mastographie \\
\hline \multirow[t]{2}{*}{ Mastoïde } & & Mastoïde \\
\hline & Mastoïdectomie & Mastoïdectomie \\
\hline Mastoïdite & Mastoïdite & Mastoïdite \\
\hline Mastologie & & Mastologie \\
\hline Mastologue & & \\
\hline
\end{tabular}


3. COMPARAISON DES ADJECTIFS COMPRIS ENTRE «PARANOÏA»ET «PARASYMPATHOMIMÉTIQUE » DANS LES QUATRE DICTIONNAIRES MÉDICAUX ÉVALUÉS

\begin{tabular}{|c|c|c|c|}
\hline GManu & PManu & Flamm & GarDe \\
\hline Paranoïaque & Paranoïaque & & paranoïaque \\
\hline Paranoïde & Paranoïde & Paranoïde & \\
\hline \multicolumn{4}{|l|}{ Paranormal } \\
\hline Paraombilical & Paraombilical & & \\
\hline \multicolumn{4}{|l|}{ Paraovarien } \\
\hline & Parapatellaire & & \\
\hline \multicolumn{4}{|l|}{ Parapéritonéal } \\
\hline Parapexien & & & Parapexien \\
\hline \multicolumn{4}{|l|}{ Paraphtisique } \\
\hline & Paraplégique & & \\
\hline Parapneumonique & & & Parapneumonique \\
\hline \multicolumn{4}{|l|}{ Parapubère } \\
\hline \multicolumn{4}{|l|}{ Pararectal } \\
\hline \multicolumn{4}{|l|}{ Pararénal } \\
\hline \multicolumn{4}{|l|}{ Parasacré } \\
\hline \multicolumn{4}{|l|}{ Parasagittal } \\
\hline \multicolumn{4}{|l|}{ Parasensible } \\
\hline Parasitaire & & Parasitaire & Parasitaire \\
\hline \multirow{2}{*}{ Parasite } & & Parasite & \\
\hline & & & Parasiticide \\
\hline \multicolumn{4}{|l|}{ Parasitifère } \\
\hline \multicolumn{4}{|l|}{ Parasitologique } \\
\hline Parasitotrope & & & Parasitotrope \\
\hline \multicolumn{4}{|l|}{ Paraspécifique } \\
\hline \multicolumn{4}{|l|}{ Parasplénique } \\
\hline Parasternal & Parasternal & & \\
\hline Parasympathicolytique & Parasympathicolytique & & Parasympathicolytique \\
\hline Parasympathicomimétique & & & Parasympathicomimétique \\
\hline Parasympathique & Parasympathique & & Parasympathique \\
\hline Parasympatholytique & Parasympatholytique & & \\
\hline parasympathomimétique & parasympathomimétique & Parasympathomimétique & \\
\hline
\end{tabular}

